\title{
3-D Flash Lidar Performance in Flight Testing on the Morpheus Autonomous, Rocket-Propelled Lander to a Lunar-Like Hazard Field
}

\author{
Vincent E. Roback ${ }^{1}$, Farzin Amzajerdian ${ }^{1}$, Alexander E. Bulyshev², Paul F. Brewster ${ }^{1}$, and Bruce W. \\ Barnes $^{1}$
}

\author{
1. NASA Langley Research Center, Hampton, Virginia 23681, USA
}

2. Analytical Mechanics Associates, Inc., Hampton, Virginia 23666, USA

\begin{abstract}
For the first time, a 3-D imaging Flash Lidar instrument has been used in flight to scan a lunar-like hazard field, build a 3-D Digital Elevation Map (DEM), identify a safe landing site, and, in concert with an experimental Guidance, Navigation, and Control (GN\&C) system, help to guide the Morpheus autonomous, rocket-propelled, free-flying lander to that safe site on the hazard field. The flight tests served as the TRL 6 demo of the Autonomous Precision Landing and Hazard Detection and Avoidance Technology (ALHAT) system and included launch from NASA-Kennedy, a lunar-like descent trajectory from an altitude of $250 \mathrm{~m}$, and landing on a lunar-like hazard field of rocks, craters, hazardous slopes, and safe sites $400 \mathrm{~m}$ down-range. The ALHAT project developed a system capable of enabling safe, precise crewed or robotic landings in challenging terrain on planetary bodies under any ambient lighting conditions. The Flash Lidar is a second generation, compact, real-time, air-cooled instrument. Based upon extensive on-ground characterization at flight ranges, the Flash Lidar was shown to be capable of imaging hazards from a slant range of $1 \mathrm{~km}$ with an $8 \mathrm{~cm}$ range precision and a range accuracy better than $35 \mathrm{~cm}$, both at $1-\sigma$. The Flash Lidar identified landing hazards as small as 30 $\mathrm{cm}$ from the maximum slant range which Morpheus could achieve $(450 \mathrm{~m})$; however, under certain wind conditions it was susceptible to scintillation arising from air heated by the rocket engine and to pre-triggering on a dust cloud created during launch and transported down-range by wind.
\end{abstract}

Keywords: 3-D Imaging, Laser RADAR, ALHAT, Asteroid, Flash Lidar, Lunar Landing, Mars, Morpheus, Precision Navigation, Safe Landing

\section{INTRODUCTION}

Landing mission concepts being developed for the exploration of planetary bodies increasingly require precision landings on sites of high scientific value making onboard, real-time terrain hazard detection and avoidance capabilities a necessity. Future human exploration missions will similarly require precision landing with increased levels of safety over those performed in the Apollo program if they are to become more commonplace. Despite the successes of the Apollo program, which was constrained to land under favorable lighting conditions at sites with no significant terrain challenges, two of the six missions experienced near disaster during the landing phase with all six landings described as being perilous. ${ }^{1}$ As an example, Figure 1 shows that Apollo 15 landed partially in a crater which resulted in a crumpled main engine bell and a vehicle resting attitude near the safe limit. Landing hazards have affected recent Mars missions requiring them to land far from the sites of primary scientific interest for fear of terrain, requiring the rovers to undertake months- or years-long drives before being able to begin the key science investigations. 


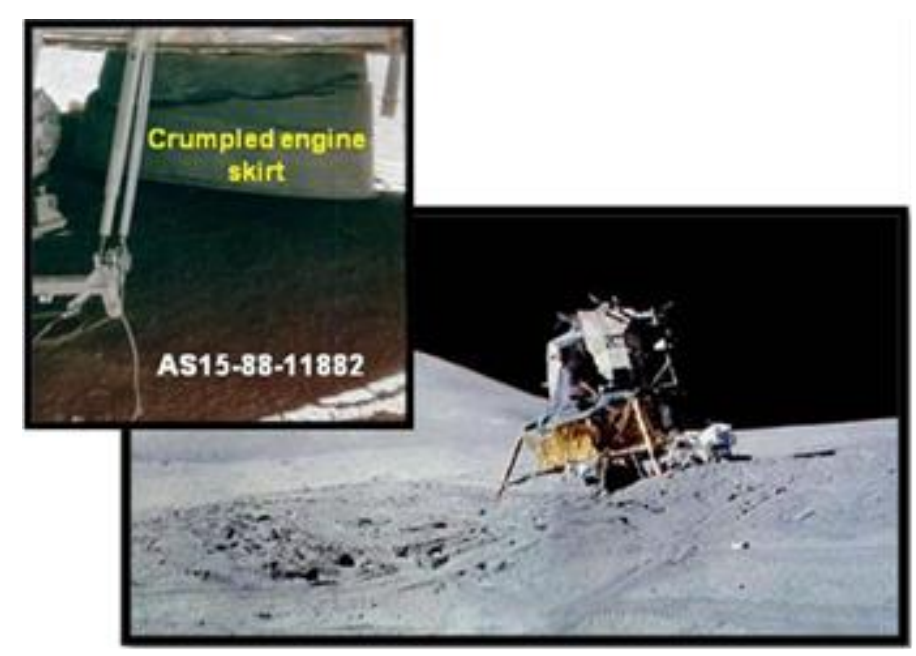

Figure 1. Apollo 15 landed partially in a crater which crumpled its main engine nozzle and left the vehicle in a resting attitude near its safe limit. ${ }^{1}$

The Autonomous Precision Landing and Hazard Detection and Avoidance Technology (ALHAT) project, led by NASA Johnson Space Center, was established by NASA to develop and demonstrate a guidance, navigation, and control (GN\&C) and sensing system for future planetary landing missions capable of terrain hazard avoidance (see Figure 2) and precision landing under any lighting conditions anywhere on the body. ${ }^{2}$ The ALHAT system is to enable the safe, precise landing for both crewed and autonomous landers. To meet the requirements of providing global access to a planetary body (moon, Mars, asteroid, etc.) under any lighting conditions, ALHAT is pursuing active Lidar sensor technology development and maturation to implement the following sensor functions: altimetry, velocimetry, Terrain Relative Navigation (TRN), Hazard Detection and Avoidance (HDA) and Hazard Relative Navigation (HRN). The Flash Lidar is being considered for performing all of the required sensor functions with the exception of velocimetry for which a Navigation Doppler Lidar (NDL) is being developed. ${ }^{3,4}$ The ability of the NDL to provide velocity data with a precision better than $0.2 \mathrm{~cm} / \mathrm{sec}$ is highly attractive for precision landing. Additionally, the NDL provides high resolution altitude and ground-relative attitude data that may further improve precision navigation to the identified landing site. The Laser Altimeter (LA) provides independent altitude data over a large operational altitude range of 20 $\mathrm{km}$ to $100 \mathrm{~m}$. All three laser sensors have a planned nominal update rate of $30 \mathrm{~Hz}$. The functions and operational range goals of each of the three ALHAT Lidar sensors have been previously reported. ${ }^{5}$

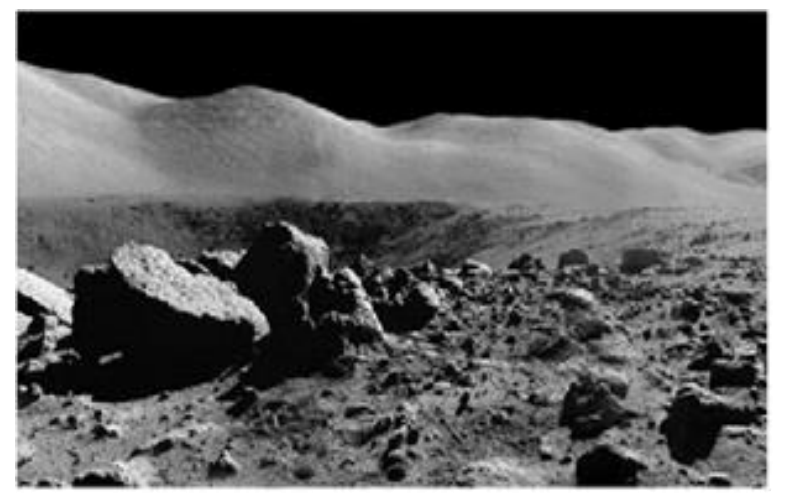

Figure 2. Future planetary landing missions (moon shown here) are considering scientifically interesting sites which happen to be near craters and rough terrain.

The Flash Lidar produces three-dimensional (3-D) images of surfaces and hazards at video rates and, as such, it is being evaluated for use as the primary landing system sensor. ${ }^{5}$ An imaging Flash Lidar system records a 3-D image of a scene by converting intensity versus time of flight of short laser pulses into intensity versus distance along the line-of- 
sight (LOS) for each spatially resolved area within a two-dimensional (2-D) image. In older, more conventional imaging lidar systems, each 2-D pixel is recorded with a separate laser pulse. Thus many laser pulses are required to record large, multi-pixel images. A Flash Lidar system records full 3-D images with a single laser pulse, permitting higher data rates and freezing-out movement within the scene and motion of the transmitter/receiver platform. The need for high speed raster scanners to sequentially address image pixels is also eliminated. The receiver is much like the familiar digital camera, but with "smart pixels" that are capable of recording the required sequential temporal information. The sensor's desired range precision is $5 \mathrm{~cm}$ at $1 \sigma$ and its desired spatial precision also known as its Ground Sample Distance (GSD) is $10 \mathrm{~cm}$ at a slant range of $750 \mathrm{~m}$. The correlation between desired range and spatial precision and its ability to resolve actual landing hazards as small as $40 \mathrm{~cm}$ has been previously reported. ${ }^{6}$ The range precision is the statistical agreement of each of the Flash Lidar's 16,384 pixels on the range to an effectively flat target (all should ideally report the same range). GSD is the ground footprint of one pixel on a target at normal incidence. The GSD and range precision levels required are intuitively clear since measurement precision must generally be several times better than the minimum desired quantity to be measured (which, in the present case, is an image range variation which points to the presence of a hazard) especially given that the hazard detection is accomplished autonomously without the aid of human interpretation.

Each of the three ALHAT sensors play a role in the ALHAT operational concept as shown in Figure 3 and previously reported. ${ }^{5}$ The POST-2 simulation was utilized extensively to optimize the ALHAT GN\&C architecture, sensor algorithms, and sensor hardware. Data from each of the three sensors is ultimately fed into a navigation filter to enhance the real-time knowledge of the vehicle navigation state (which can be in error by hundreds of meters due to Inertial Measurement Unit drift during travel time from Earth) relative to the ground to enable precise navigation to and landing on the optimal safe site nearest to the originally intended mission landing site. The Flash Lidar executes its HDA and HRN functions as the sensor portion of the ALHAT Jet Propulsion Laboratory (JPL) Hazard Detection System (HDS). ${ }^{7}$ The $\mathrm{HDA} / \mathrm{HRN}$ functions require detection of rocks and surface features greater than $40 \mathrm{~cm}$ in height, detection of slopes greater than $5^{0}$ over the diagonal of the footprint of the landing vehicle, and determination of landmark position to better than one meter relative to the specified landing location. The HDA concept employed in the present field test campaign involves scanning the landing site with the largest Field-of-View (FOV) which will still meet the spatial resolution goals and stitching the images together in a mosaic to create a 3-D DEM of the landing site in near-real-time. The HRN concept employed in the present field test campaign included the use of subsequent Flash Lidar images after the initial DEM was created in order to track and navigate relative to a feature (typically another hazard and hence the name HRN) near the safe site by identifying it on the DEM and inferring vehicle state based upon the pose difference between the initial DEM and the present images of the hazard as the vehicle moves and the hazard changes perspective. The HRN phase continues to nominally $100 \mathrm{~m}$ where the landing dust cloud can become a factor in obscuring the ground. 


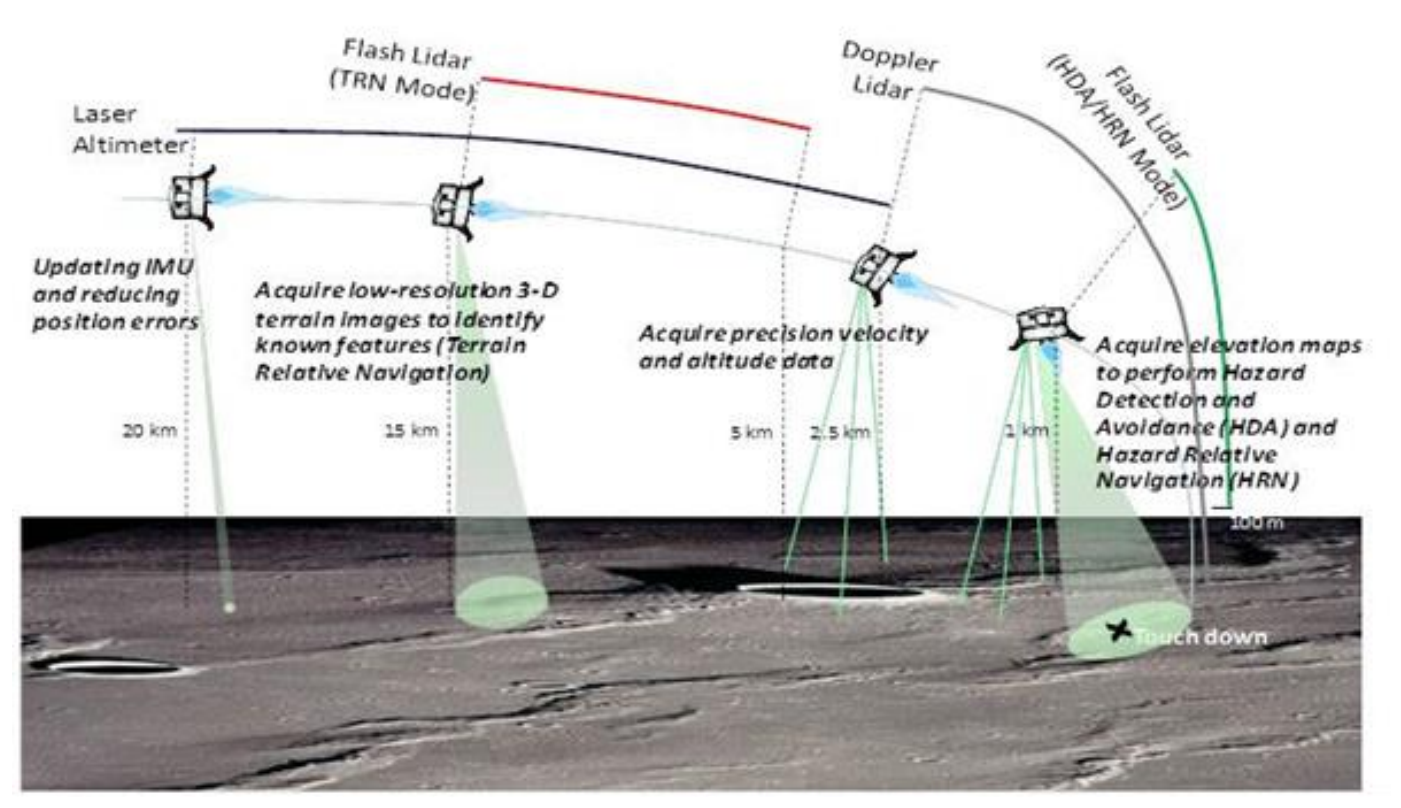

Figure 3. ALHAT concept of operations.

The three lidar sensor systems have undergone a series of development and five field test cycles over the eight year lifecycle of the ALHAT project. ${ }^{8}$ Initial results of the culminating campaign of the project, Field Test 6 (FT6), have recently been reported. ${ }^{8,9}$ The present report will provide greater detail on the Flash Lidar results. With the demonstration in a relevant environment during FT6, the ALHAT system reached its TRL 6 goal. For FT6, the ALHAT system was integrated to the Morpheus rocket-propelled, free-flying test bed and flown on a lunar-like approach trajectory to landing on the same lunar-like hazard field at KSC that was used in the FT5 helicopter testing. FT6 marked the first time that the ALHAT system was used in a closed-loop fashion to scan a lunar-like hazard field, identify a safe landing site, and autonomously help to guide a vehicle to a safe landing on the hazard field. The campaign consisted of three daytime open-loop flights, one daytime closed-loop flight, and one nighttime closed-loop flight all following the same trajectory which peaked in altitude at $250 \mathrm{~m}$ at a slant range to the hazard field of approximately $500 \mathrm{~m}$ and moving downrange from the launch site to the hazard field approximately $400 \mathrm{~m}$. The flights were monitored from the Morpheus Mission Control Center (MMCC) with telemetry data from the sensors displayed at the ALHAT console. The Flash Lidar executed its HDA objective by generating 3-D range images in real-time at a level of precision which enabled hazards as small as $30 \mathrm{~cm}$ to be identified from the maximum slant range provided by Morpheus. The HDS then stitched together a mosaic of the $1^{\circ}$ FOV Flash Lidar images to build a 3-D DEM of the hazard field in near-real-time and successfully identify all five safe sites amongst the hazardous rocks, craters, and slopes and passed the preferred safe site on to the Morpheus GN\&C system. The Flash Lidar executed its HRN function following HDA and the HDS fed the position updates into the ALHAT navigation filter for precision guidance of Morpheus over portions of its trajectory to the safe site. The NDL provided high-fidelity velocity updates to the ALHAT navigation filter over portions of the trajectory for precision guidance to the selected safe site. The Laser Altimeter fed slant range data to the ALHAT navigation filter for precision guidance to the selected safe site. Each of the three sensors experienced minor anomalies due to air heated by the rocket plume. In the end, the ALHAT system reached its TRL 6 goal through successful demonstration in a relevant environment.

\section{FLASH LIDAR SYSTEM CONFIGURATION}

The 3-D imaging Flash Lidar built by NASA-Langley (LaRC) is a second generation (Gen 2.2), compact, real-time, air-cooled, autonomous, $20 \mathrm{~Hz}$ time-of-flight sensor system based on 3-D imaging IR camera technology developed by Advanced Scientific Concepts (ASC). ${ }^{10}$ The Flash Lidar sensor system, with specifications delineated in Table 1, consists of two boxes as shown in Figure 4: the Flash Lidar Sensor Head (FLSH) and the Flash Lidar Electronics Box 
(LEB). A full series of thermal and vibration environmental testing was accomplished based on the expected Morpheus environments. The FLSH integrates a $1.064 \mu \mathrm{m} 50 \mathrm{~mJ}$ class IV Neodymium: Yttrium Aluminum Garnet (Nd:YAG) Fibertek pulsed laser ( $8 \mathrm{~ns}$ Full-Width-Half-Max or FWHM pulse width) operating at $20 \mathrm{~Hz}, 1^{\circ} \mathrm{FOV} 100 \mathrm{~mm}$ f/7.3 receiver optics, $1^{\circ}$ divergence transmitter optics, and a Indium Gallium Arsenide-Avalanche Photodiode (InGaAs-APD) detector hybridized to a Read-Out Integrated Circuit (ROIC) which together constitute the 128 pixel by 128 pixel focal plane array (FPA) residing in an ASC Tiger Eye camera. Tiger Eye serial number 1004 with a fresh FPA of appropriate sensitivity and nearly devoid of dead pixels $(<1.1 \%$ of its total pixel count) was utilized for the Morpheus testing as opposed to the equivalent sensitivity Tiger Eye 1005 camera and FPA used in the previous UH-1H testing of 2012 (FT5) which had accumulated a dead pixel count of $7.1 \%$ due to humidity exposure to its unsealed FPA (unsealed since it was still experimental). ${ }^{11}$ Other changes since the FT5 testing included replacement of the $1^{\circ}$ compound receiver lens with a lens of equivalent FOV and throughput which did not require the single element corrective lens required by the existing one, slight increase to the divergence of the transmitter optics to increase the overfill factor for robustness to transmitter/receiver misalignment, and addition of dry-gas purge ports to the FLSH and LEB to preclude methane fume build-up from the Morpheus tank off-gassing and to provide protection from the Texas and Florida humidity. A new series of pointing metrology tests were conducted to map the unit vectors from the FPA pixels through the receiver optics to a set of fiducials hard-mounted to the FLSH frame which were in turn mapped to the navigation frame of the ALHAT system. The Flash Lidar receiver optics are bore-sighted with a small optical witness camera (Go Pro Hero 3 with a custom lens) mounted to the FLSH belly which provides a larger ( $8^{\circ}$ along the diagonal) FOV context for the LIDAR image. The LEB houses the laser electronics unit, the PC-104-based sensor controller (programmed in C++ with a Linux operating system), a Vicor-based power conditioning and distribution subsystem, signal conditioning and thermal control circuitry, and a laser termination system for safety which switches off the laser power supply when commanded remotely via telemetry. To provide for terrestrial operations in humid environments, the Flash Lidar has provisions for dry-gas purge during all hangar and flight operations. The addition of a dry-gas (CO2) purge bottle to Morpheus during the summer of 2014 provided purge gas during the last remaining periods where purging was not possible previously, i.e. the time just before, during, and just after flight.

The inputs to the Flash Lidar include 28 VDC power, time synchronization signals, and commands. The Flash Lidar outputs real-time calibrated, corrected 3-D range images with a range precision of $8 \mathrm{~cm}(1-\sigma)$, an absolute range accuracy of better than $35 \mathrm{~cm}$ over the operational range, and the necessary $10 \mathrm{~cm} \mathrm{GSD}$ at a $750 \mathrm{~m}$ slant range to allow reliable detection of hazards smaller than the $40 \mathrm{~cm}$ goal. A subset of the data is output via a serial port for telemetry and display in mission control. Several real-time signal processing steps are applied to the raw images, including application of the precision calibration, application of the range accuracy calibration, masking of dead or intermittent pixels, application of a five-point median filter, insertion of a header which contains various house-keeping data parameters, and application of an Automatic Gain Correction (AGC) function. The AGC adjusts detector voltage (and, thereby, sensitivity) autonomously in order to maintain the image intensity within the narrow dynamic range of the sensor in order to produce images at the desired range precision level by operating within the range/intensity calibration. Based on lessons learned in the FT5 testing in 2012, additional tailoring was incorporated into the AGC settings to preclude instabilities in image intensity during AGC-commanded changes (i.e. ensuring the intensity changes made by AGC were small in comparison to the upper and lower thresholds selected which would trigger and AGC-commanded change).

The Flash Lidar performance as stated in Table 1 meets the HDA/HRN goals (the goals under test presently) which were set early in the ALHAT project with the exception of the range precision goal of $5 \mathrm{~cm}$. However, the $8 \mathrm{~cm}$ range precision proved more than sufficient to detect hazards smaller than the $40 \mathrm{~cm}$ resolution goal. The GSD of Table 1 is better than the ALHAT goal at the maximum slant range which Morpheus could provide since the Flash Lidar was designed to achieve the GSD goal at a larger slant range of 750m, thus at smaller slant ranges the GSD improves given the fixed-FOV receiver optics. 


\section{Flash Lidar Sensor Head}

(FLSH)

Flash Lidar Electronics Box

Mounted to HDS 2-axis

Gimbal

(LEB)

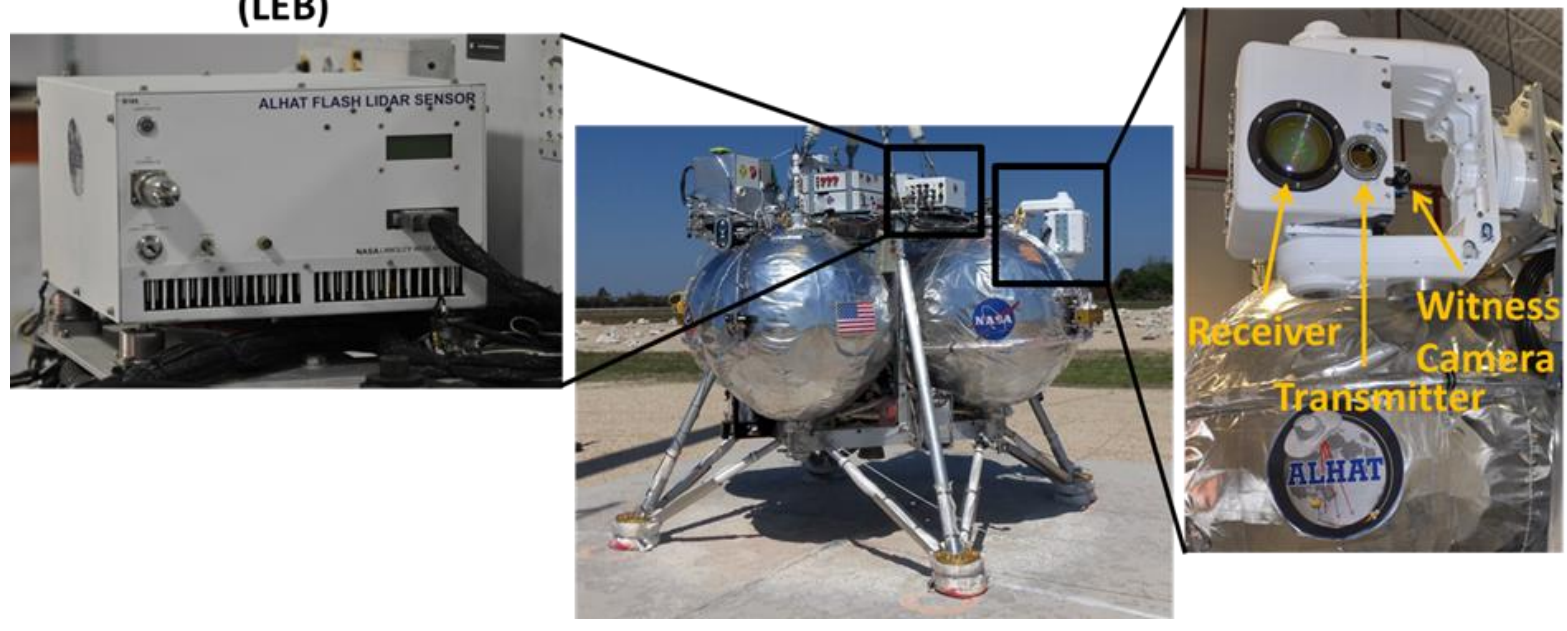

Figure 4. Generation 2.2 Flash Lidar sensor system consisting of Flash Lidar Sensor Head (FLSH) at right and Flash Lidar Electronics Box (LEB) at left mounted to the Morpheus rocket with the KSC lunar hazard field in the background.

Table 1. Flash Lidar sensor specifications.

\begin{tabular}{|c|c|c|}
\hline \multicolumn{2}{|c|}{ Parameter } & Value \\
\hline \multicolumn{2}{|l|}{ Max operational range } & $1.3 \mathrm{~km}^{(1,2)}$ \\
\hline \multirow{3}{*}{ Min operational range } & Defocus Limit & $250 \mathrm{~m}$ (2 pixel defocus) \\
\hline & \multirow{2}{*}{ Saturation Limit } & $150 \mathrm{~m}^{(2)}$ \\
\hline & & $100 \mathrm{~m}^{(3)}$ \\
\hline \multicolumn{2}{|l|}{ Range precision } & $8 \mathrm{~cm}(1-\sigma)$ \\
\hline \multicolumn{2}{|l|}{ Range accuracy } & $<35 \mathrm{~cm}(1-\sigma)$ \\
\hline \multirow{2}{*}{$\begin{array}{l}\text { Ground footprint (at } 430 \mathrm{~m} \text { ), } \\
\text { normal target }\end{array}$} & Full FOV & $7.5 \mathrm{~m} \times 7.5 \mathrm{~m}$ \\
\hline & 1 pixel (GSD) & $5.9 \mathrm{~cm}$ \\
\hline \multirow{2}{*}{ Size } & FLSH & $11 \mathrm{Hx} 13.5 \mathrm{D} \times 13.25 \mathrm{~W}$ inches \\
\hline & LEB & $9.5 \mathrm{H} \times 14.2 \mathrm{D} \times 13 \mathrm{~W}$ inches \\
\hline \multirow{2}{*}{ Weight } & FLSH & $36 \mathrm{Ibs}^{(4)}$ \\
\hline & LEB & $36 \mathrm{Ibs}^{(4)}$ \\
\hline \multicolumn{2}{|l|}{ Power } & 450 Watts $^{(4)}$ \\
\hline
\end{tabular}

${ }^{1} 30^{\circ}$ line-of-sight

${ }^{2} 70 \%$ normal reflectivity at $1.064 \mu \mathrm{m}$

${ }^{3} 30 \%$ normal reflectivity at $1.064 \mu \mathrm{m}$

${ }^{4}$ Dominated by terrestrial environmental control subsystem

\section{LAB CHARACTERIZATION RESULTS}

Prior to flight, the Flash Lidar was extensively characterized at a LaRC outdoor test facility referred to as the Long Distance Test Range (LDTR) which allowed testing at ranges to be experienced during the Morpheus campaigns. The test range was utilized to assess maximum operational range as well as to calibrate and characterize 
ultimate range precision and range accuracy performance. Table 1 details the lab performance results, which met all of the performance requirements laid out in the introduction.

The LaRC LDTR is shown in overview in Figure 5. It consists of a fixed trailer 860m from the large B1261 target building, a fixed tent $760 \mathrm{~m}$ from B1261, a road which extends from the tent up to B1261 (to provide for any intermediate ranges desired to B1261), and a set of small target boards at set distances from the tent used in range accuracy calibrations. A truck in which the lidar was mounted, was driven along the road between the tent and the B1261 in order to vary the range to B1261 for the range precision calibration and verification testing. Figure 6 shows the B1261 target building. In addition to its sets of square and hemispherical targets (painted for a normal reflectivity of $30 \%$ at $1.064 \mu \mathrm{m}$ to match the sand reflectivity at NASA-Armstrong where previous developmental flight testing was conducted), B1261 contains a flat area bare of any target in its upper right quadrant which is useful for range precision testing. A set of five range accuracy target boards (20 inches by 20 inches) were located at set distances for use in range accuracy calibration.

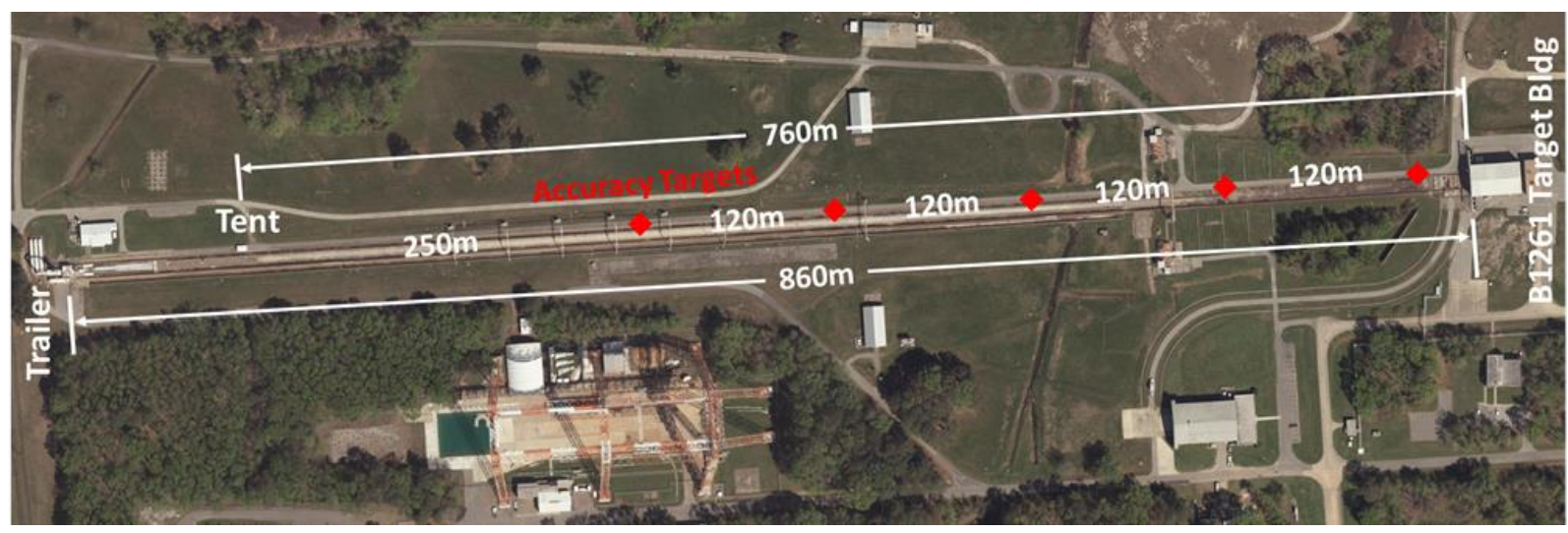

Figure 5. LaRC Long Distance Test Range (LDTR) showing relative positions of lidar trailer, lidar tent, five accuracy calibration targets (positioned at ranges from the tent of 250m, 370m, 490m, 610m, and 730m), road along which truck can be operated, and large B1261 target building.

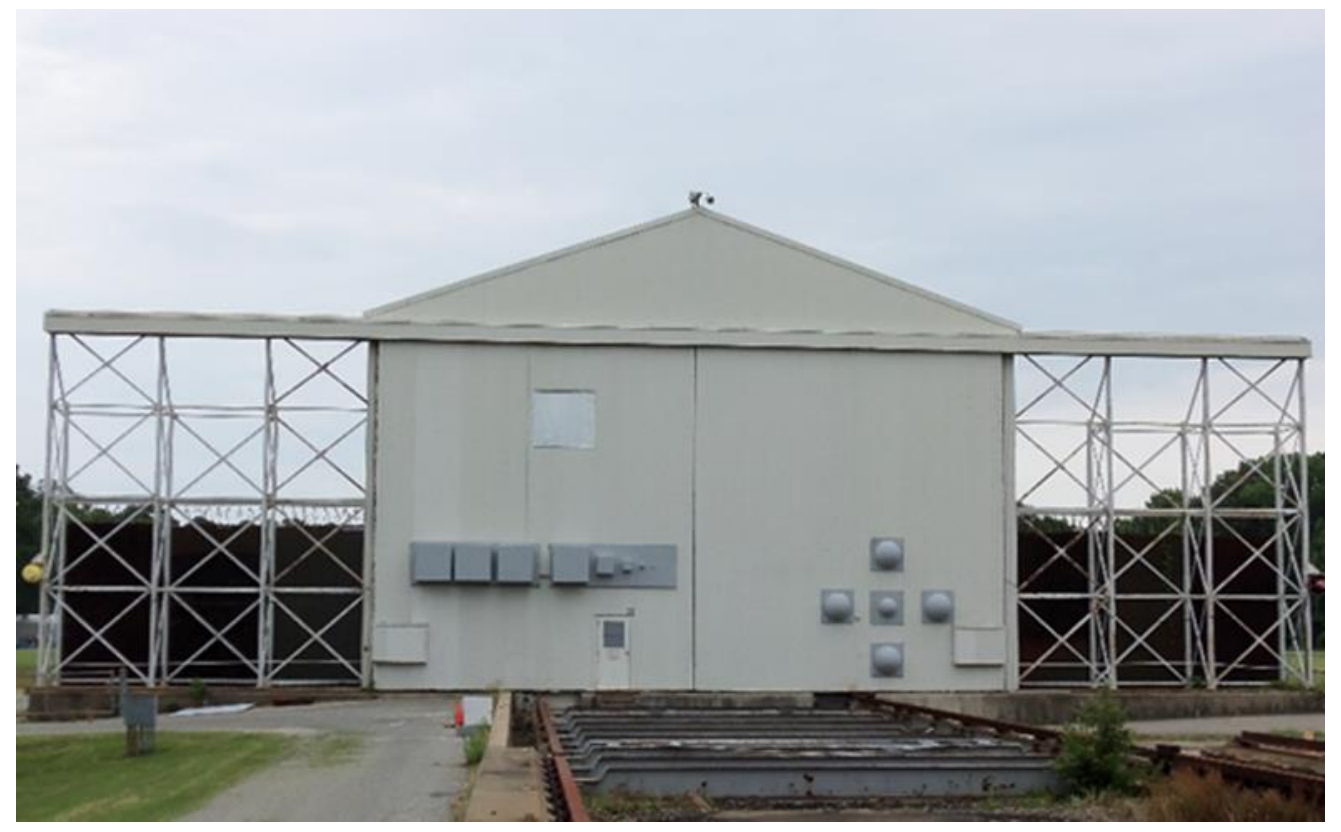

Figure 6. LaRC LDTR B1261 target building. 
The maximum operational range of the Flash Lidar was characterized on the LDTR B1261 target building with the Flash Lidar in its full flight configuration (as described in the configuration section above). Based upon extrapolations from the LDTR results (shown in Figure 7) to the flight conditions, the predicted maximum operational slant range is approximately $1,300 \mathrm{~m}$ at the $30^{\circ}$ line-of-sight approach angle planned $\left(60^{\circ}\right.$ angle of optical incidence). Given that the maximum slant range between the lidar and the KSC hazard field in the planned Morpheus Hazard Detection Profile (HDP) trajectory is $500 \mathrm{~m}$, the $1,300 \mathrm{~m}$ performance is more than sufficient.

The extrapolation from LDTR results to flight predictions was accomplished by comparing the present LDTR results with those from past years which had been previously correlated to flight conditions during the 2012 FT5 campaign of reference 11. The reflectivity of the bare wall portions of B1261 (i.e. the regions free of the rectangular cuboid targets, hemispherical targets, small window, and small door) at normal incidence was confirmed to be equivalent to the reflectivity of the KSC hazard field at the $30^{\circ}$ line-of-sight angle $\left(60^{\circ}\right.$ angle of optical incidence) at which the lidar views the hazard field from the maximum slant range portion of the Morpheus trajectory. The reflectivity of the KSC hazard field at normal $\left(0^{\circ}\right)$ incidence for $1.064 \mu \mathrm{m}$ was determined to be approximately $70 \% \pm 10 \%$.
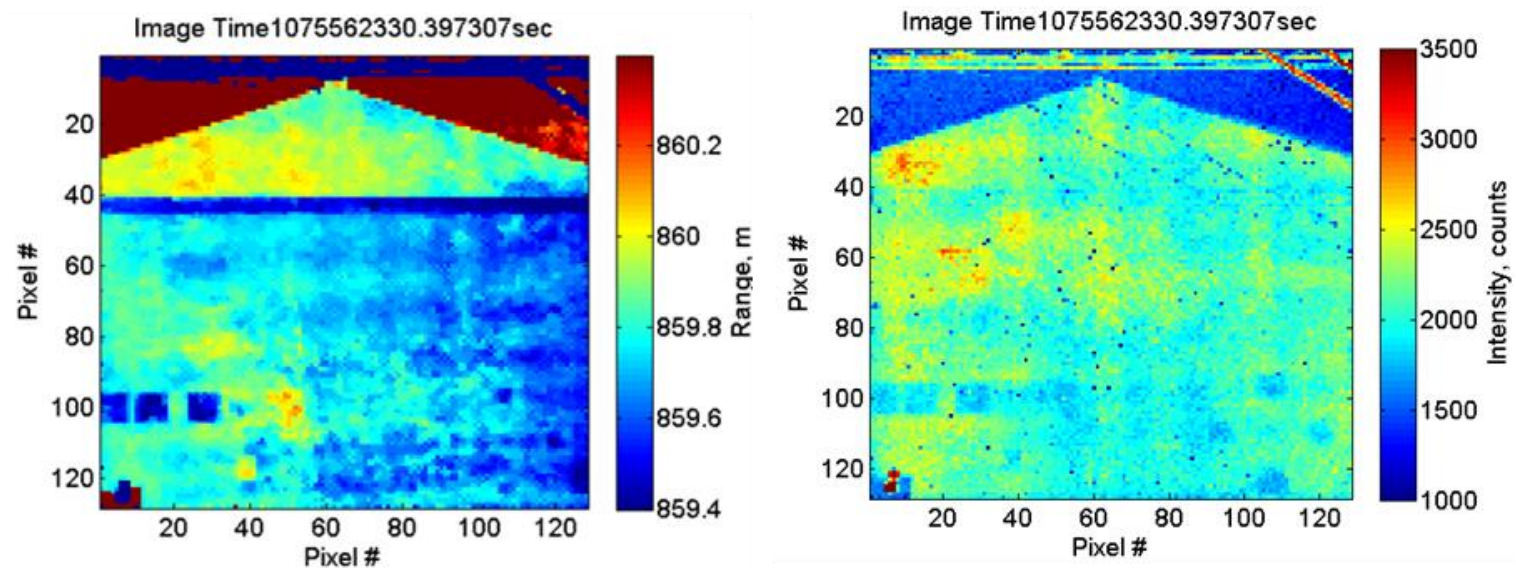

Figure 7. Maximum operational range performance on LaRC LDTR B1261 target building, February 4, 2014.

A series of range precision verification tests were conducted on the LaRC LDTR to confirm the quality of the range and intensity calibration tests also conducted on the LDTR. An $8 \mathrm{~cm}$ range precision $(1 \sigma)$ is achieved across the image when the range/intensity calibration is applied. Range precision is essentially range noise on an image that can serve to hide a hazard which has a size comparable to the noise level. As a reminder, algorithm simulations indicate that a $5 \mathrm{~cm}$ range precision coupled with a $10 \mathrm{~cm}$ or smaller GSD is needed in order to reliably resolve $30 \mathrm{~cm}$ hazards. ${ }^{6}$ Range precision statistically quantifies the agreement on range when all pixels are presented with a flat target and is defined as one standard deviation $(1 \sigma)$ among the 16,384 pixels within a single frame of range data. Without the calibration, the range precision degrades to approximately $30 \mathrm{~cm}(1 \sigma)$. Range precision is measured by imaging a flat portion of the B1261 target building at approximately normal incidence (so that each pixel should see approximately the same range to target) from ranges which approximately correspond to the maximum and minimum slant ranges of the Morpheus trajectory over which the Flash Lidar is utilized. In order to obtain the desired range from the B1261 target building, the lidar and its support equipment is mounted in a truck with a lidar window installed in its front and parked at the appropriate point on the LDTR road that leads up to B1261. The upper right portion of the target building (from the orientation of a person facing the building) is utilized since it is flat and free of targets and surface features. Insertion of neutral density (ND) filters over the beam output provides the attenuation needed in order to vary return intensities over the dynamic range of the sensor.

Figure 8 shows the range precision from the calibration performance testing at $300 \mathrm{~m}$ and $600 \mathrm{~m}$. Range precision is plotted as a function of average return intensity over a given image. Figure 8 a shows that at $300 \mathrm{~m}$, the $8 \mathrm{~cm}$ goal is achieved from roughly 1800 counts up through 3500 counts, with a degradation in range precision below 1800 counts which is attributable to a low signal-to-noise ratio in which many pixels are not triggered and thus produce a false range. Figure $8 \mathrm{~b}$ at $600 \mathrm{~m}$ shows similar results, but with data points only available to 3000 counts given the longer range to target and accompanying weaker signal returns. 


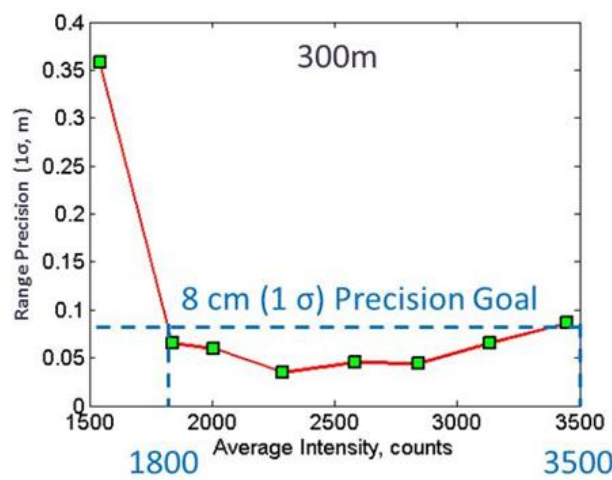

(a) $300 \mathrm{~m}$

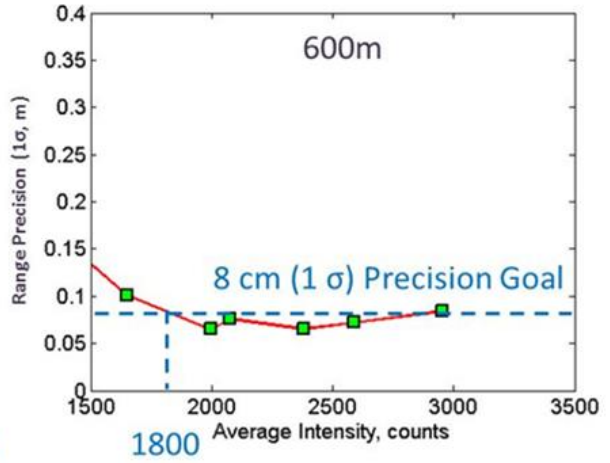

(b) $600 \mathrm{~m}$

Figure 8. Range precision results for 300m and 600m ranges from LaRC LDTR B1261 target building upper right flat area on December 5, 2013 which shows the subset of intensities over which the sensor achieves the project's goals.

Figure 9 provides a sample of the range and intensity calibration performance (range precision) at one particular intensity value (i.e. at one of the data points plotted in Figure 8a). Figure 9a shows the intensity image of the upper right portion of B1261. Figure 9a also displays the associated histogram which indicates that the intensity was generally uniform over the image. Figure $9 \mathrm{~b}$ shows a range image of that upper right portion of B1261, note the general agreement on range of all of the pixels. Figure $9 \mathrm{~b}$ also shows the associated range histogram to further illustrate that the 16,384 pixels generally agree tightly on the range to target. Figure 10 provides the same illustration as Figure 9 except at $600 \mathrm{~m}$ rather than $300 \mathrm{~m}$. Since Figure 10 is at a larger range, more of B1261 is visible given the fixed FOV of the receiver optics. Thus, the upper right of the image should be considered since it is the flat, feature-free portion of the building which can give insight into the range precision performance of the Flash Lidar. Again in Figure 10b (as in Figure 9b), the pixels tightly agree on range to target illustrating the overall performance shown in Figure 8. 


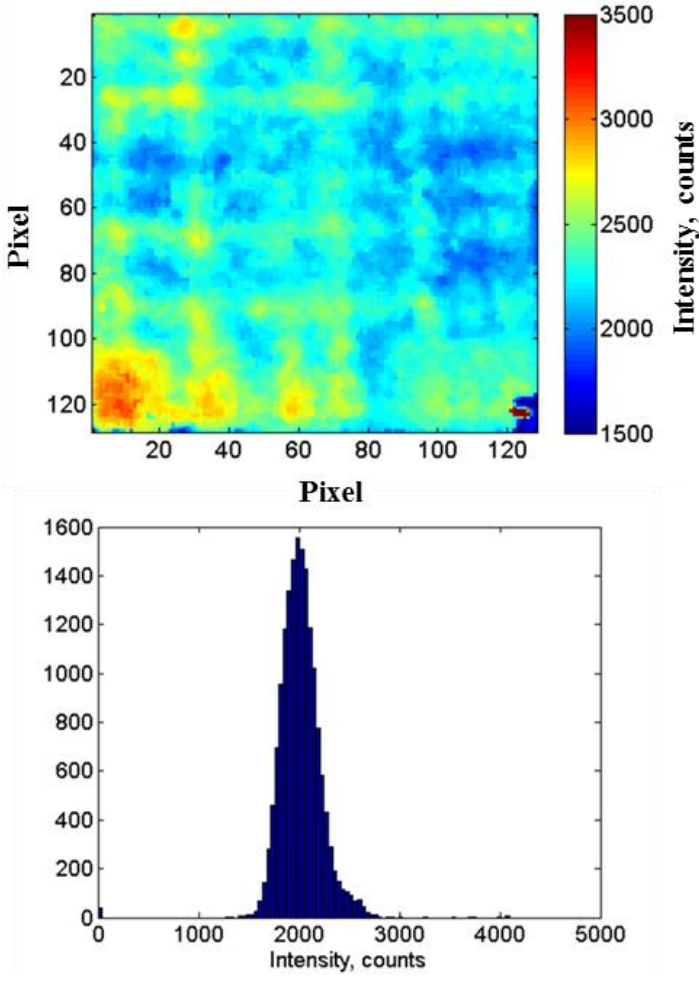

(a) Intensity
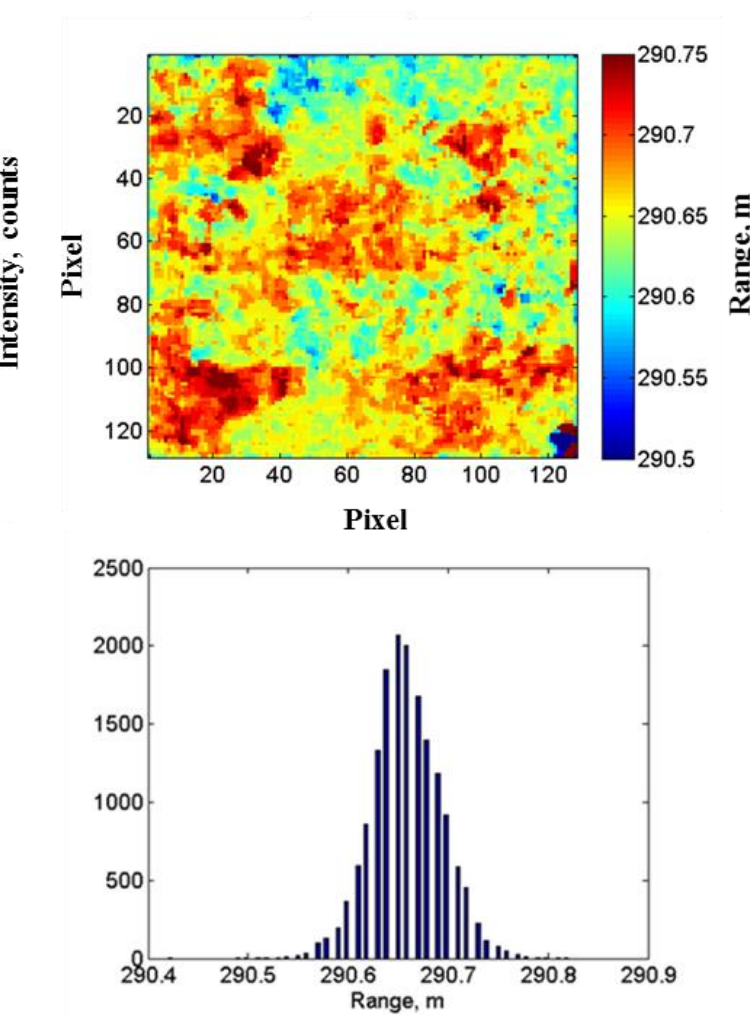

(b) Range

Figure 9. Range and intensity calibration performance at 300m on the LDTR B1261 target building upper right flat region, December 5, 2013.

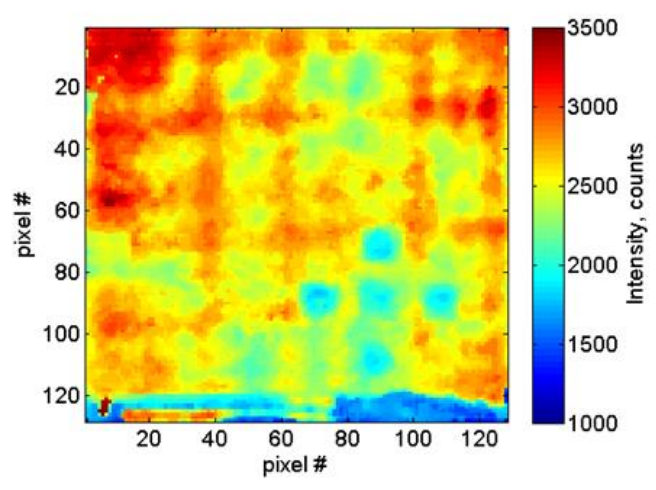

(a) Intensity

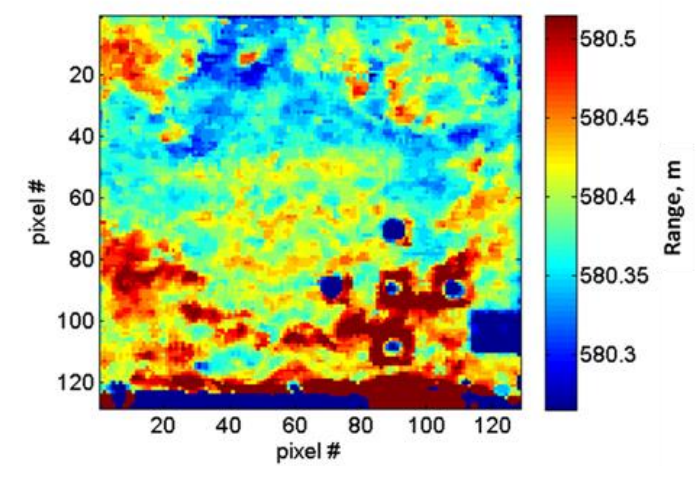

(b) Range

Figure 10. Range and intensity performance at $600 \mathrm{~m}$ on the LDTR B1261 target building upper right flat region, December 5, 2013.

A series of range accuracy calibration and verification tests were conducted on the LaRC LDTR. The Flash Lidar was situated in the tent, see Figure 5, on the LDTR and it imaged a set of five small 20 inch by 20 inch target boards situated at ranges to cover the operational range of the Flash Lidar $(250 \mathrm{~m}, 370 \mathrm{~m}, 490 \mathrm{~m}, 610 \mathrm{~m}$, and $730 \mathrm{~m})$, as shown in Figure 5. The reflectivity at $1.064 \mu \mathrm{m}$ of each target was selected depending upon its range to the lidar such that the return intensity of all targets would fall within the dynamic range of the lidar so that they could all be analyzed within the same frame. Analysis of all targets within the same image frame minimized the range noise on each of the five 
measurements (caused by image-to-image uncertainties) which improved the curve fit fidelity and yielded more accurate linear calibration coefficients. The location of the five targets was surveyed to a $2 \mathrm{~cm}$ accuracy and a range resultant was calculated to give known ranges from the Flash Lidar sensor to each of the targets. A set of linear range accuracy calibration coefficients were computed based upon actual range to the targets versus the Flash-Lidar-reported range over several iterations to obtain convergence. To check the final quality of the calibration, the five targets were imaged again to check for accuracy performance, however, unlike the calibration runs, the performance runs included image-to-image uncertainties. Range error for a given target was computed by subtracting the surveyed range resultant to the target from the lidar's reported range to the target. The lidar's reported range to the target was computed by taking the mean of all pixels on the target over 200 frames. The error bars of Figure 11 indicate plus and minus one standard deviation of the error at each target which is mainly due to image-to-image variability, but which also includes surveying errors as well as the range precision limit of the Flash Lidar. The slight slope visible in the error plot of Figure 11 was not corrected by adjusting the calibration coefficients given the tight schedule constraints of the project and since the error met requirements over the Morpheus slant ranges for which the lidar would be relied upon $(250 \mathrm{~m}$ to $500 \mathrm{~m})$ to provide accuracy range values to the ALHAT navigation filter. The range accuracy performance was also spot checked during Morpheus pre-flight, on-ground operations at KSC during pointing tests in which surveyed targets were imaged at ranges representative of those to be flown. Overall the Flash Lidar range error is better than $35 \mathrm{~cm}(1 \sigma)$ for the ranges tested between $250 \mathrm{~m}$ and $730 \mathrm{~m}$.

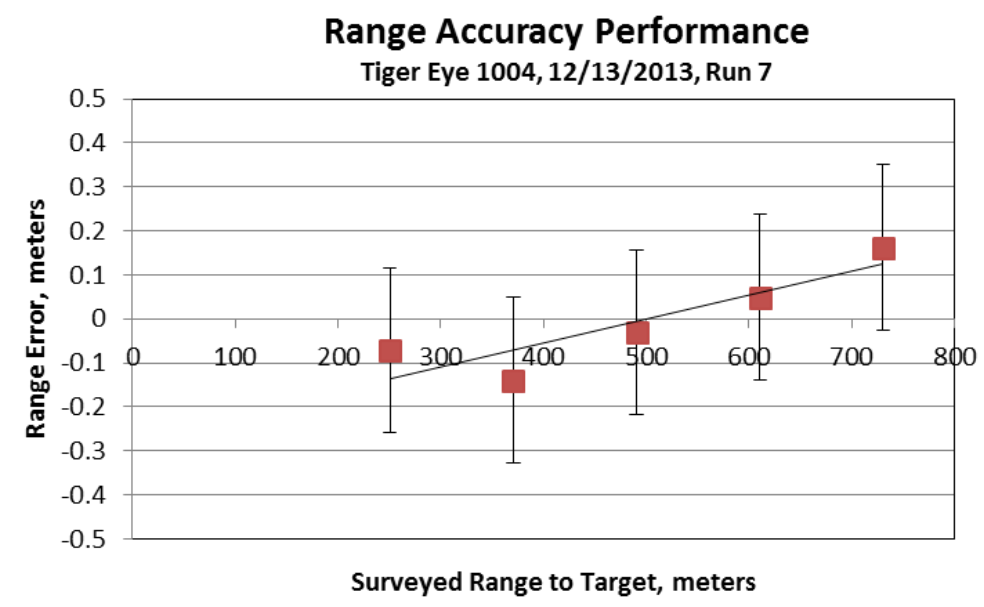

Figure 11. Tiger Eye 1004 range accuracy performance from 12/13/2013 as measured on the LaRC LDTR in which five target boards fixed normal to the lidar at surveyed ranges were imaged.

\section{MORPHEUS INTEGRATION AND FLIGHT TEST PLAN}

The FT6 flight test campaign which occurred in the spring and fall of 2014 was conducted onboard the Morpheus 1.5B free-flying rocket-propelled lander flying approaches to the KSC lunar-like hazard field. The three lidars along with the other portions of the ALHAT GN\&C system (partners at JPL and JSC) were integrated to Morpheus at KSC. During the prior summer, an integration and tether test campaign had been conducted at JSC on the $1.5 \mathrm{~B}$ vehicle for the purpose of identifying and correcting system integration issues. During the same interval, the ALHAT sensors underwent a series of minor upgrades based upon lessons learned from the JSC tether testing. A single tether test was conducted at KSC to confirm that all systems remained nominal before attempting the first integrated ALHAT/Morpheus free flight. The FLSH was hard-mounted to the 2-axis HDS gimbal which was in turn hard mounted to the Morpheus upper deck. The hard mounting ensured minimal attitude uncertainty relative to the vehicle navigation center. The LEB was mounted to the upper deck of Morpheus via four shock-vibration isolators.

The Morpheus rocket-propelled, free-flying test-bed was built by JSC using a design based upon the Armadillo Pixel vehicle. It is powered by a gimbaled engine burning a mixture of Liquid Oxygen (LOX) and Methane. The LOXMethane propulsion system, still a highly experimental propulsion system, was chosen since its fuel could be manufactured in-situ using compounds present in planetary bodies and also since its fuel is clean-burning and does not produce pollutants. Morpheus also utilizes a hot-gas LOX-Methane Reaction Control System (RCS) for vehicle attitude 
adjustments in flight. The LOX-Methane systems, like the ALHAT systems, are part of the technology development portion of the project. Although the vehicle operated autonomously, the MMCC, located on the bottom floor of the Shuttle Landing Facility (SLF) air traffic control tower, issued various pre-flight commands and received extensive telemetry for real-time monitoring of its various subsystems (including the ALHAT systems) using the Integrated Test and Operations System (ITOS). The MMCC also housed the range safety officer station which was able to issue the laser safety termination command for the Class IV non-eye-safe Flash Lidar as well as both the soft abort (vehicle lands immediately and autonomously) and hard abort (engine shuts down and the vehicle falls) commands in the event that the vehicle strayed outside of its safety bounds.

The ALHAT FT6 campaign onboard Morpheus consisted of single approaches (one per flight) to a lunar-like hazard field at KSC as shown in Figure 12. The Hazard Detection Profile (HDP) trajectory reached $245 \mathrm{~m}$ in altitude and extended $405 \mathrm{~m}$ downrange. The launches occurred at the north end of the former SLF runway 33 and the landings occurred at the safe-site on the lower right of the KSC lunar-like hazard field. In the hours leading up to launch, the combined performance of the ALHAT navigation system, the HDS gimbal pointing system, and the Flash Lidar systems are tested by commanding pointing to two targets at fixed, surveyed points at ranges of approximately $90 \mathrm{~m}$ and $350 \mathrm{~m}$. When the combined system was able to point to and image the targets, confidence was gained in system configuration and performance for the impending flight. During the open-loop test flights, the Morpheus navigation system was in control of the vehicle and the ALHAT system functioned as it would if it were commanding the vehicle except that Morpheus would not accept the ALHAT guidance. During the closed-loop flights, the Morpheus navigation system would begin accepting vehicle guidance from ALHAT early in the descent portion of the trajectory at which point ALHAT would identify the safe-site and continue to provide navigation updates to Morpheus for precision guidance to that safe site. If the ALHAT guidance deviated outside of a tight tolerance set by the Morpheus, then guidance would revert back to the Morpheus GN\&C inputs. Note that the tolerances set by Morpheus were necessarily tighter than those on which the ALHAT GN\&C was based for actual lander missions given the experimental nature of Morpheus and its inability to land under moderate off-nominal conditions safely.

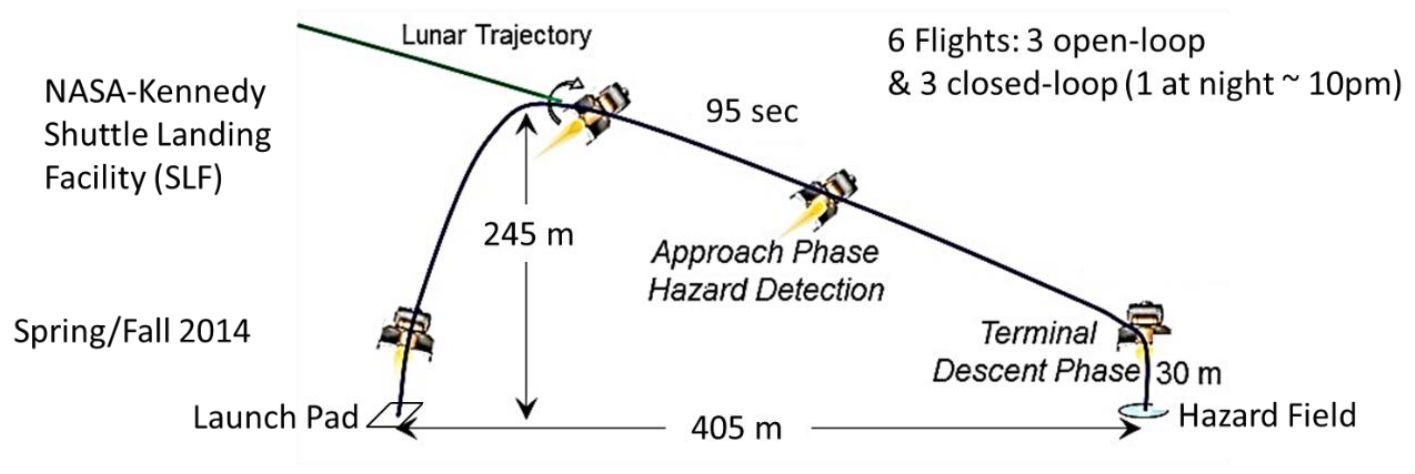

Figure 12. Morpheus HDP trajectory flown during the spring/fall 2014 FT6 campaign for FF10 - FF15. The HDP included launch at the north end of the SLF runway 33 and landing on the custom-built lunar-like hazard field.

The KSC lunar-like hazard field, shown in Figure 13, is a 100m by $100 \mathrm{~m}$ custom-built field consisting of hazardous craters, rocks, and slopes representative of actual lunar distributions as well as safe sites. Each hazard feature and safe site was precision surveyed. A reference truth DEM was generated based upon the hazard field high-fidelity survey information for comparison against the in-flight generated ALHAT DEM's. 

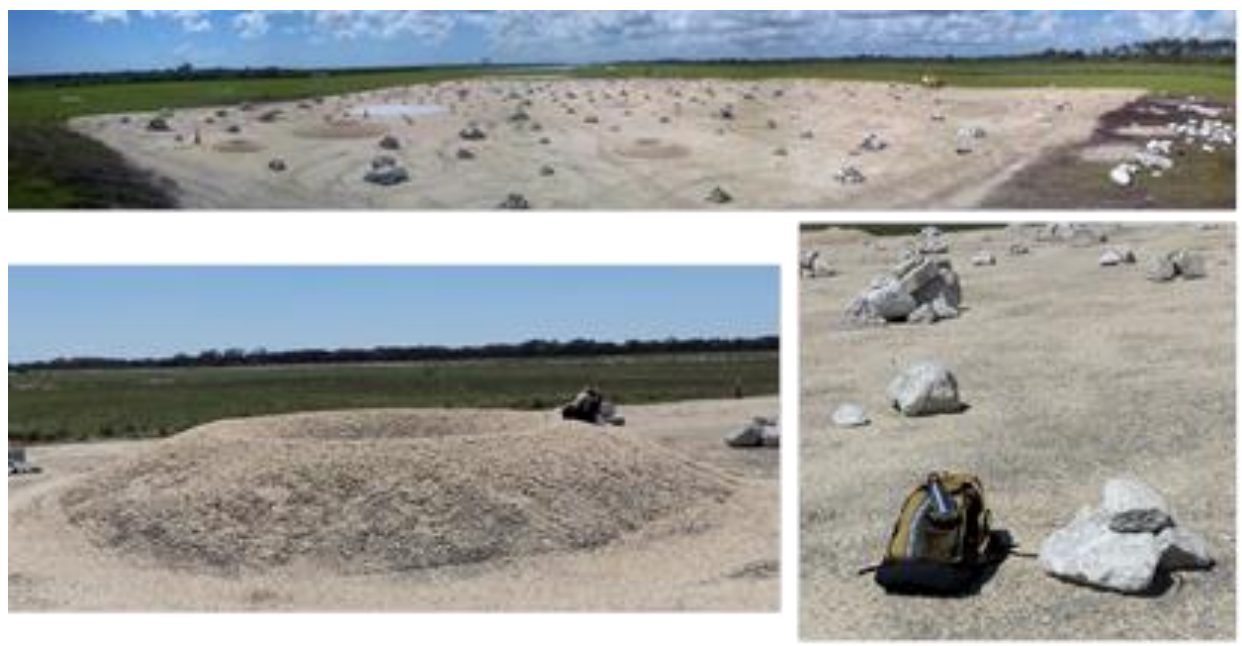

Figure 13 . KSC hazard field $100 \mathrm{~m}$ by $100 \mathrm{~m}$ in size containing rock (heights ranging from 30 to $100 \mathrm{~cm}$ ) and crater hazards along with hazardous slopes and safe sites located off the north end of the KSC SLF runway.

\section{FLIGHT TEST RESULTS}

The Flash Lidar, in concert with the NDL and LA lidar sensors and the experimental GN\&C system, provided the performance needed in order to execute successful closed-loop, autonomous free-flights in which the Morpheus rocketpropelled, free-flying test bed was guided by ALHAT through its trajectory to a safe site on the lunar-like hazard field at KSC. A total of six free-flights (three open-loop flights and three closed-loop flights) were completed from April 2 December 15, 2014 and are summarized in Table 2. The sensors provided data to not only identify the safe site amongst the field of hazardous craters, rocks, and slopes in both day and night lighting conditions, but also provided updates to the ALHAT navigation filter in order to enable precision guidance of Morpheus by the ALHAT navigation system to a safe site. Each of the three sensors experienced occasional performance degradation due to the Morpheus environment, however, not generally at the same time which permitted the hazard detection and guidance to proceed successfully. The chief source of performance degradation occurred under certain wind conditions as the beams of the sensors passed through air heated by the rocket engine which created scintillation. The Flash Lidar also suffered from pre-triggering under certain wind conditions which transported a dust cloud created during launch down-range into its FOV. After some minor changes to the NDL and LA during the summer of 2014 and a restriction on winds for the Flash Lidar, FF15 was flown free of degradation. 
Table 2. Flight log for FT6 free-flight testing at KSC.

\begin{tabular}{|c|c|c|c|c|}
\hline Flight \# & Type & Date & $\begin{array}{c}\text { Launch } \\
\text { Time } \\
\text { GMT } \\
\text { (EDT+4) }\end{array}$ & Dec \\
\hline FF10 & Open-loop flt \#1 & $\begin{array}{c}\text { Wed, } \\
04 / 02 / 2014\end{array}$ & $20: 22$ & 94 \\
\hline FF11 & Open-loop flt \#2 & $\begin{array}{c}\text { Thurs, } \\
04 / 24 / 2014\end{array}$ & $19: 22$ & 96 \\
\hline FF12 & Open-loop flt \#3 & $\begin{array}{c}\text { Wed, } \\
04 / 30 / 2014\end{array}$ & $17: 57$ & 92 \\
\hline FF13 & Closed-loop flt \#1 & $\begin{array}{c}\text { Thurs, } \\
5 / 22 / 2014\end{array}$ & $18: 29$ & 94 \\
\hline FF14 & $\begin{array}{c}\text { Closed-loop flt \#2, } \\
\text { Night flt \#1 }\end{array}$ & $\begin{array}{c}\text { Wed, } \\
5 / 28 / 2014\end{array}$ & $2: 02$ & 96 \\
\hline FF15 & Closed-loop flt \#3 & $\begin{array}{c}\text { Mon, } \\
12 / 15 / 2014\end{array}$ & $21: 11$ & 94 \\
\hline
\end{tabular}

\footnotetext{
Morpheus Free-Flying Rocket-Powered Test Bed with ALHAT Payload Airport: NASA-Kennedy Shuttle Landing Facility (SLF) KTTS Runway 15-33 Hazard field: Located at departure end of NASA-Kennedy SLF runway 33 FF: Free-flight (not tethered to crane)
}

The calibrated and corrected 3-D range images generated autonomously and in real-time by the Flash Lidar were stitched together into a near-real-time DEM by the HDS and processed to correctly find five safe sites on all flights under the HDA objective. ${ }^{9}$ The safest site was correctly ranked by the HDS on all flights and passed to the Morpheus navigation system for landing during the closed-loop flights. On one closed-loop flight, the safe site was rejected by Morpheus because of a half-meter offset in the ALHAT-selected landing point (Flash Lidar scintillation induced by air heated by the Morpheus rocket engine and an internal HDS mapping anomaly were contributing factors) even though it was within the ALHAT landing criteria for a spacecraft lander; the Morpheus landing criteria was necessarily tighter due to its experimental nature. The HDS-selected safe site was rejected on another closed-loop flight because the safe site selected by HDS happened to not be one which had the structural reinforcement necessary to bear the weight of Morpheus. During that flight, one of the reinforced safe sites had been populated with an array of small rocks for a piggy-back experiment which may have been detected by the ALHAT system consequently lowering the rank of that safe site and causing it to not be selected as the safest of the five options. After mosaic generation and safe site selection, the Flash Lidar images continued to be used for generation of navigation updates under the HRN objective. The FlashLidar-based HRN updates that were accepted by the navigation filter provided fine tuning of the navigation solution and contributed to precision navigation as part of the closed-loop GN\&C. The Flash Lidar met its performance requirements which in turn enabled the HDS to successfully accomplish its mission. The Flash Lidar met its operational range requirements, successfully generating range images from the maximum slant range down to the minimum needed. Although the Flash Lidar fell just short of its $5 \mathrm{~cm}$ range precision goal and instead achieved $8 \mathrm{~cm}$, its calibrated and corrected images were more than sufficient to enable detection of hazards $30 \mathrm{~cm}$ in size and larger, thereby exceeding the original ALHAT hazard detection goals. 


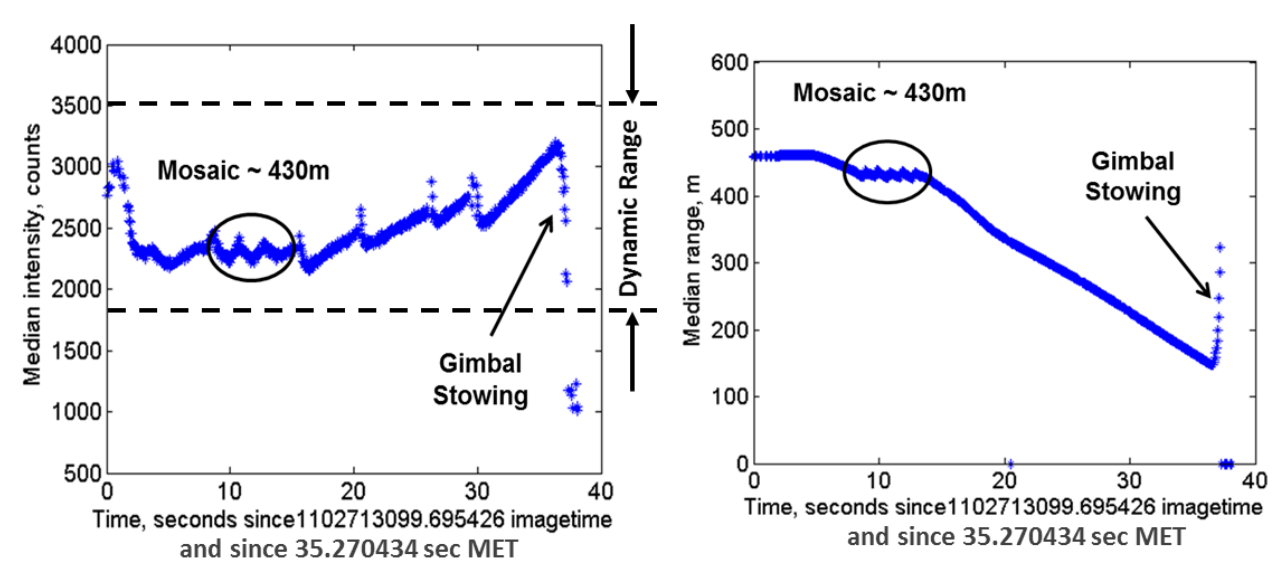

(a) Median Intensity

(b) Median Slant Range

Figure 14. Median intensity (a) and median slant range (b) profile for the latter portion of FF15.

The Flash Lidar generated 3-D images of the hazard field from the maximum slant range allowable by Morpheus' current generation engine of approximately $470 \mathrm{~m}$ at a $30^{\circ} \mathrm{LOS}$ angle (resulting in a $60^{\circ}$ angle of incidence for the Lidar) down to below the minimum slant range requirement of $250 \mathrm{~m}$. Previous flight testing onboard a UH-1H helicopter to the same KSC hazard field validated the Flash Lidar's maximum operational slant range to be $1,350 \mathrm{~m} .{ }^{11}$ Based upon data from early 2014, the reflectivity at $1.06 \mu \mathrm{m}$ of the hazard field at an incidence angle of zero is $70 \%$ which is a contributing factor to the Flash Lidar's ability to exceed its maximum operational range requirement of $750 \mathrm{~m}$ for a $30 \%$ reflective surface. Figure 14 shows the median intensity and median slant range as a function of time for the latter portion of free-flight 15 (FF15). Image time is based upon the 1980 GPS epoch, while MET (mission elapsed time) is based upon the Morpheus ignition time for the given flight. The mosaic, in which successive real-time Flash Lidar images are received by the HDS and stitched together to form a larger FOV image of a significant portion of the hazard field, begins at a MET of approximately $44 \mathrm{sec}$ and lasts approximately 6 seconds. Figure 14a shows that the AGC function within the Flash Lidar sensor controller was consistently able to adjust detector voltage (and thereby its sensitivity) in order to maintain the image intensity within the narrow dynamic range of the sensor. Operation within the sensor's dynamic range enabled production of images at the desired range precision level of $8 \mathrm{~cm}(1-\sigma)$ so that hazards as small as $30 \mathrm{~cm}$ could be sensed. Once Morpheus descends to a slant range within 150m of the hazard field, the AGC is not able to keep the image from saturating. The consequence of image saturation is merely a small increase in range noise as range precision degrades slightly from the desired level. Only one image acquired during the flight was corrupted and it occurred outside of the mosaic. The single corrupted image was caused by a set of pixels along two extreme edges of the FOV which affected the t0 pixel due to their intermittent behavior which occurred in flight only (HDS masked these narrow columns of pixels so that they would not add noise to the range images). Figure 15 shows individual range images of the hazard field (with rocks in view) from both the maximum slant range flown on Morpheus (approximately $470 \mathrm{~m}$ ) to below the minimum range requirement of $250 \mathrm{~m}$. Each image in Figure 15 shows 128 pixels by 128 pixels of data with slant range being the contour variable. The color gradient from bottom to top in each range image is due to apparent slope caused by the $30^{\circ}$ LOS viewing angle. The minimum operational range of the Flash Lidar is dominated by saturation and defocus. Since the defocus limit is more restrictive, the Flash Lidar images are accepted for HRN processing above $250 \mathrm{~m}$. Figure 15 shows that the Flash Lidar produced images which met and exceeded the minimum range requirement. The Flash Lidar incorporates an AGC algorithm which can hold the image intensity within the sensor's dynamic range down to ranges of $150 \mathrm{~m}$ for the $70 \%$ reflectivity of the KSC hazard field. Operation within the dynamic range ensures that the range precision performance meets the $8 \mathrm{~cm}$ goal. For the reference reflectivity of $30 \%$ (closer to the typical 15\% reflectivity of the lunar surface), the Flash Lidar's minimum saturated-dominated range is $100 \mathrm{~m}$. The Flash Lidar receiver lens is set for maximum depth of field in order to provide in-focus images at all ranges above $250 \mathrm{~m}$. 


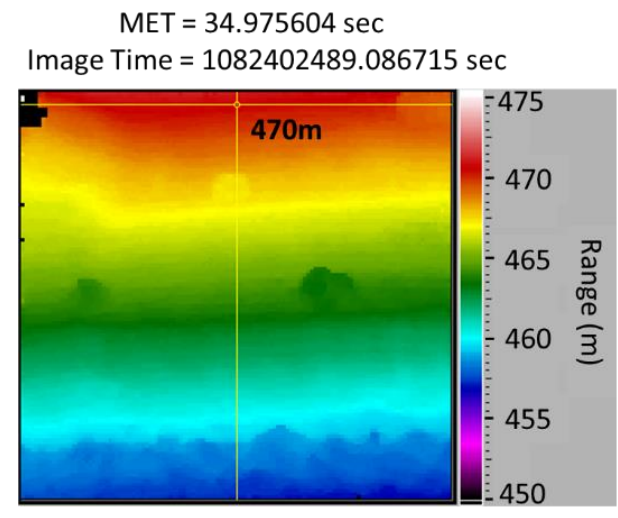

(a) Typical max slant range for Morpheus

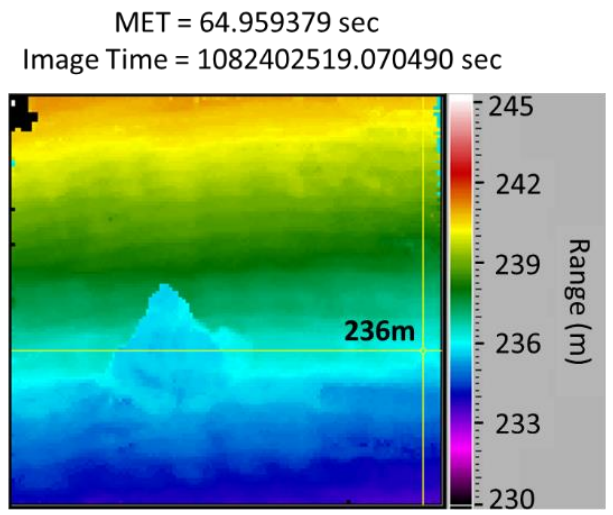

(b) Typical min slant range on Morpheus

Figure 15. Flash Lidar operational range performance $\left(30^{\circ} \mathrm{LOS}\right)$ exceeded requirements on Morpheus. The Flash Lidar was capable of larger slant ranges than Morpheus could provide. AGC held images within the calibrated dynamic range for range precision of 8 $\mathrm{cm}(1 \sigma)$ well below the $250 \mathrm{~m}$ requirement.

As an example of the hazard detection capability of the Flash Lidar, Figure 16 shows two 30cm rock hazards that are visually identifiable from a slant range of approximately $425 \mathrm{~m}$. The figure shows the surveyed ground-truth DEM described earlier of the hazard field along with an enlargement of one of the safe sites on whose periphery are the two $30 \mathrm{~cm}$ rocks of interest. Both surveyed DEM's show distance along their $\mathrm{x}$ and y axes and elevation as their contour variable. The figure also shows the same two rocks in actual Flash Lidar DEM's, each constructed from a single lidar image, whose axes and contour variable are the same as those of the truth DEM. The Flash Lidar reconstruction of the rocks shows them to be $25 \mathrm{~cm}$ in size, which is just a $5 \mathrm{~cm}$ difference from the truth DEM. The two Flash Lidar DEM's are from two flights, one a daytime flight and one a nighttime flight, which demonstrates that the Flash Lidar is equally capable in all ambient lighting conditions. For reference, the GSD at the range of Figure 16 is $6 \mathrm{~cm}$, thus better than the $10 \mathrm{~cm}$ requirement. The GSD was better than the $10 \mathrm{~cm}$ requirement since the Flash Lidar was designed to achieve a 10 $\mathrm{cm}$ GSD at 750m, thus given its fixed-FOV receiver optics, the GSD improves as slant range decreases. Data from FT5 showed that it was possible to visually identify a hazard smaller than the $40 \mathrm{~cm}$ requirement $(25 \mathrm{~cm}$ hazard height in FT5) even with a GSD worse that the $10 \mathrm{~cm}$ requirement and a range precision worse than the current $8 \mathrm{~cm} .{ }^{11}$ Thus data from both FT5 and FT6 indicate that the 40cm hazard resolution requirement has been met. Figure 17 and Figure 18 are both DEM's each created from a single Flash Lidar range image. Figure 17 shows two rock hazards and a crater hazard. Figure 18 shows the central flat area of the safe site imaged in Figure 16 and gives some insight into the low noise range images provided. The sloping to the left in the image is the natural, gradual terrain slope present in the hazard field. The Flash Lidar experienced several minor performance anomalies over the course of the campaigns. Since the launch pad is approximately $400 \mathrm{~m}$ up-range from the hazard field (i.e. launch and landing points very close by), the dust cloud created at launch was carried downrange under certain tail-wind conditions to the vicinity of the hazard field which resulted in lidar pre-triggering on the dust particles instead of on the hazard field. Wetting of the launch pad area helped to minimize the effect and a wind restriction was placed on flight operations for FF15. The Flash Lidar also experienced minor performance degradation due to scintillation. Additional details on performance degradation have been provided previously ${ }^{8}$ and will be expanded upon in a forthcoming publication. 


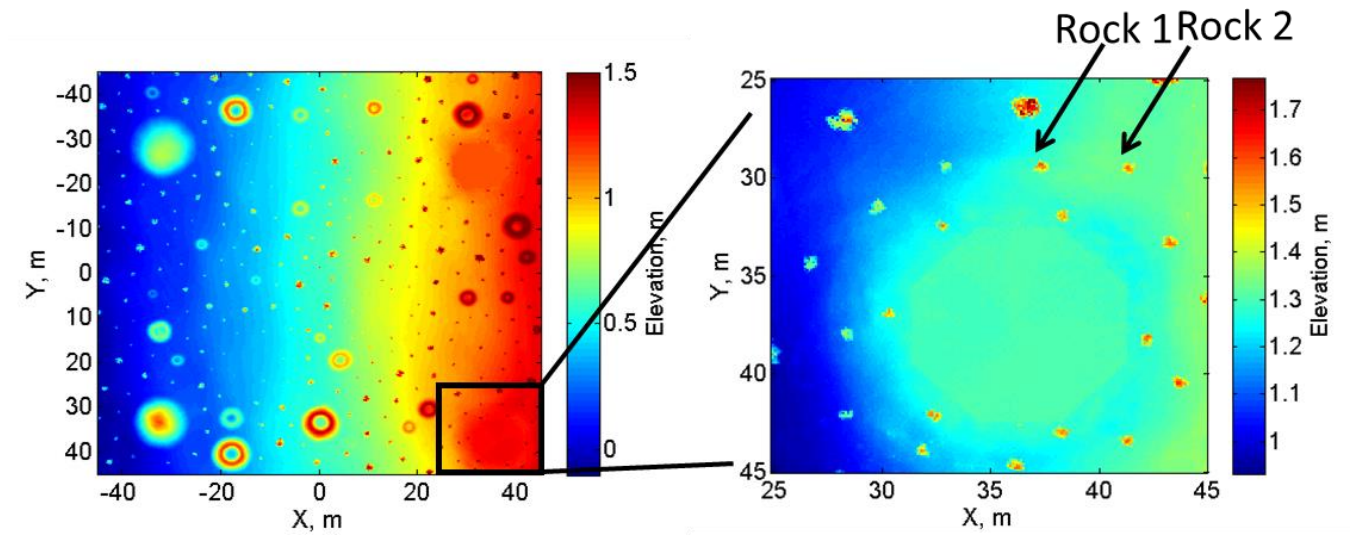

(a) Truth DEM of hazard field.

(b) Truth DEM safe site enlargement.

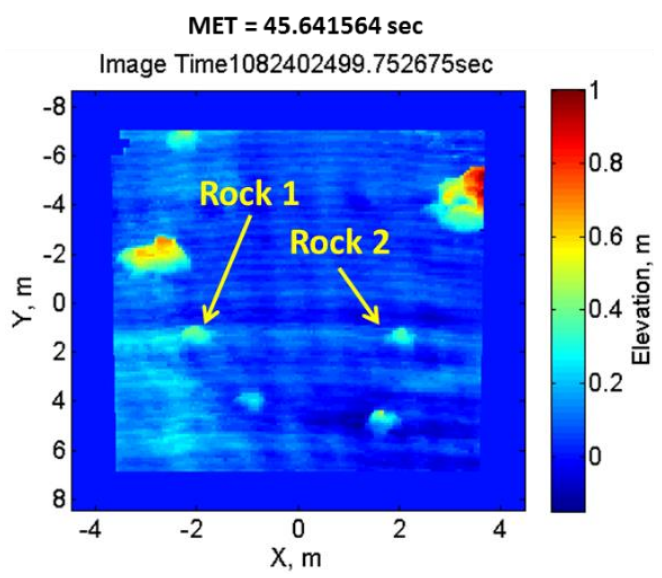

(c) Flash Lidar DEM from FF11 (day)
$\mathrm{MET}=46.036214 \mathrm{sec}$

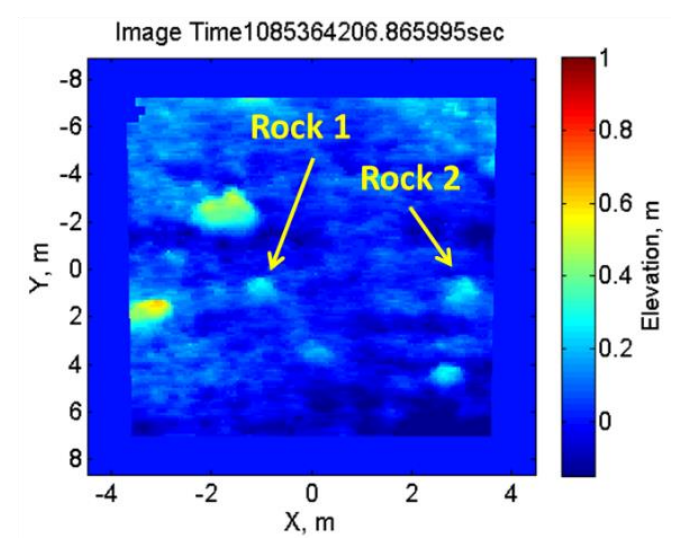

(d) Flash Lidar DEM from FF14 (night)

Figure 16. Rock hazards of $30 \mathrm{~cm}$ size (based on the truth DEM of "a" and "b") which are situated on the periphery of the safe site imaged by the Flash Lidar from approximately 425m during both day (FF11) and night (FF14) flights. Rock 1 is $30 \mathrm{~cm}$ and rock 2 is $28 \mathrm{~cm}$.

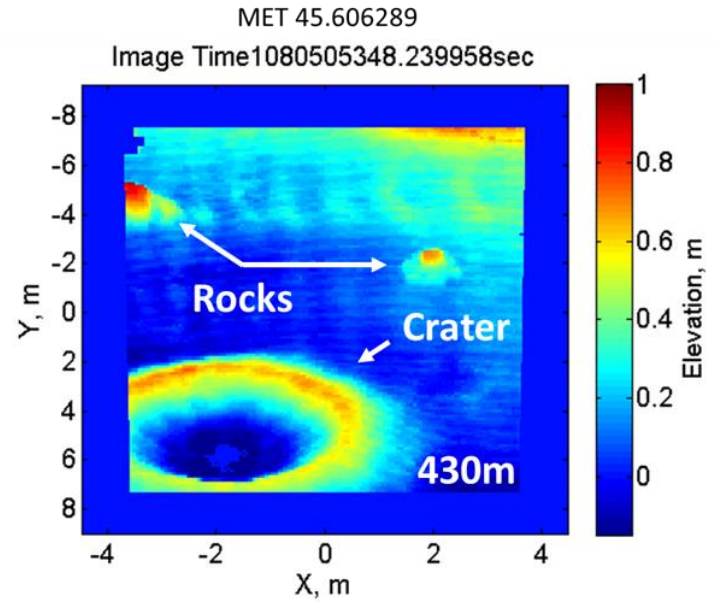

Figure 17. FF10 hazard DEM. 


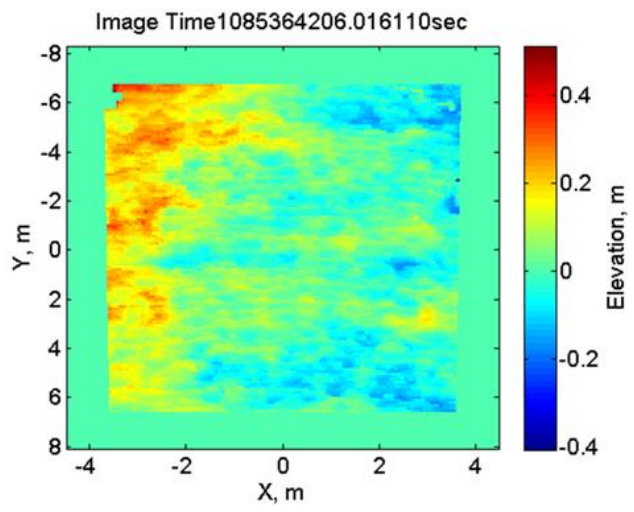

Figure 18. FF14 safe site (hazard field lower right) DEM.

\section{CONCLUSIONS}

For the first time, a GN\&C and lidar-based sensing system autonomously scanned a lunar-like hazard field from an autonomous, rocket-propelled, free-flying lander on a lunar-like approach trajectory, then correctly identified a safe site, and subsequently provided closed-loop precision guidance for landing on a safe site. An ALHAT-like system will be a necessity for future missions which will send landers to increasing complex sites (poorly lit, challenging terrain, etc.) on differing planetary bodies (moon, Mars, asteroids) due to the high scientific and mission payoff possible and will require them to do so with higher fidelity and safety. Such a technology is being pursued by many space-faring nations since it promises to end the days of needing to land far from sites of high scientific value or sites near critical in-situ resources needed for colonization for fear of hazardous terrain or poor lighting conditions. Flash Lidar technology is being evaluated by NASA to serve as the primary landing sensor in the GN\&C system for future robotic and crewed landers because of its ability to generate 3-D images of the potential landing site in real-time for use in identifying hazardous craters, rocks and sloped terrain and for its ability to provide 3-D images from high altitude (>15 km) for use in terrain matching in order to fix the navigation state during initial powered descent.

A Flash Lidar sensor system, which has been refined through several generations of development and a series of five previous field test campaigns, has been lab characterized and flight tested. The ALHAT system consisting of the Gen 2.2 Flash Lidar, the NDL, the LA, and the experimental GN\&C system accomplished its sixth field test campaign in the spring and fall of 2014. FT6 was conducted onboard the Morpheus 1.5B vehicle at KSC with landings on a custom, lunar-like hazard field located just off the north end of the SLF runway for the TRL 6 demonstration in a relevant environment. In on-ground laboratory characterization, the Flash Lidar was shown to achieve all of the sensor performance goals defined early in the ALHAT project (with the exception of range precision which was missed by $3 \mathrm{~cm}$ at $1-\sigma)$. In actual flight, the performance was shown to be more than sufficient to identify the safe site and provide precision guidance. The Flash Lidar's real-time 3-D range images at $20 \mathrm{~Hz}$ enabled the ALHAT HDS to create a 3-D DEM in near-real-time, to correctly identify hazards as small as $30 \mathrm{~cm}$ (better than the $40 \mathrm{~cm}$ ALHAT goal), to pass the correct safe site on to Morpheus, and to continue providing precision guidance to the safe site.

The ALHAT system accomplished three open-loop flights and three closed-loop flights on a trajectory that peaked at an altitude of $250 \mathrm{~m}$ and proceeded $405 \mathrm{~m}$ down range providing nearly $500 \mathrm{~m}$ of slant range for sensor testing during the approach trajectory. To prove the all-lighting performance of ALHAT, one of the closed-loop flights was accomplished in the pitch darkness of night. The Flash Lidar performance degradation initially experienced under certain wind conditions which brought air heated by the rocket engine into its FOV causing scintillation and which brought a dust cloud created at launch down-range into its FOV causing pre-triggering was mitigated through a wind restriction on flight operations. The ALHAT system autonomously identified the safe landing site and provided closedloop, precision guidance for landing on a safe site to enable the TRL 6 demonstration.

\section{ACKNOWLEDGEMENTS}


The authors would like to express appreciation to the ALHAT project manager, Chirold Epp of the NASA-Johnson Space Center, for his invaluable support. The authors are thankful to Advanced Scientific Concepts, Inc. for providing the Flash Lidar camera and for supporting the static and airborne tests. The authors acknowledge the ALHAT team members from the NASA-Jet Propulsion Laboratory for their collaboration on the HDS. The authors are thankful to the ALHAT and Morpheus team members at the NASA-Johnson Space Center for all of their efforts and support including the provision of the Morpheus flight vehicle without which ALHAT would have had few options for demonstrating its autonomous hazard detection and precision guidance capabilities. We would like to acknowledge Bill Carrion and Anna Noe for their support as master technicians and Gregory Gaddis for facilitating the field test at NASA-Kennedy Space Center. We also would like to thank NASA's Advanced Exploration Systems (AES) program office for their continued support.

\section{REFERENCES}

[1] Brady, T., and Paschall, S., "The Challenge of Safe Lunar Landing," Proceedings of the IEEE Aerospace Conference, IEEE, Piscataway, NJ, 1-14, (2010).

[2] Epp, C.D., Robinson, E.A., and Brady, T., "Autonomous Landing and Hazard Avoidance Technology (ALHAT)", Proceedings of the IEEE Aerospace Conference, IEEE, Piscataway, NJ, 1-7, (2008).

[3] Amzajerdian, F., Pierrottet, D., Tolson, R. H., Powell, R. W., John B. Davidson, and Peri, F., "Development of a Coherent Lidar for Aiding Precision Soft Landing on Planetary Bodies," Proceedings of the $13^{\text {th }}$ Coherent Laser Radar Conference, Kamakura, Japan, (2005).

[4] Pierrottet, D., Amzajerdian, F., Petway, L., Barnes, B., and Lockard, G., "Flight test performance of a high precision navigation Doppler lidar," Proceedings of SPIE, Vol. 7323, SPIE, Bellingham, WA, 11-1-11-9, (2009).

[5] Amzajerdian, F., Pierrottet, D. F., Petway, L. B., Hines, G. D., Roback, V. E., and Reisse, R. A., "Lidar Sensors for Autonomous Landing and Hazard Avoidance," AIAA 2013-5312, (2013).

[6] Huertas, A., Johnson, A. E., Werner, R. A., Maddock, R. A., "Performance Evaluation of Hazard Detection and Avoidance Algorithms for Safe Lunar Landings," Proceedings of the IEEE Aerospace Conference, IEEE, Piscataway, NJ, 1 - 20, (2010).

[7] Carson, J. M., Bailey, E. S., Trawny, N., Johnson, A. E., Roback, V. E., Amzajerdian, F., and Werner, R. A., "Operations Concept, Hardware Implementation and Ground Test Verification of a Hazard Detection System for Autonomous and Safe Precision Lunar Landing," AAS 13-856, (2013).

[8] Roback, V. E., Pierrottet, D. F., Amzajerdian, F., Barnes, B. W., Bulyshev, A. E., Hines, G. D., Petway, L. B., Brewster, P. F., and Kempton, K. S., "Lidar Sensor Performance in Closed-Loop Flight Testing of the Morpheus RocketPropelled Lander to a Lunar-Like Hazard Field," AIAA 2015-0328, (2015).

[9] Trawny, N., Huertas, A., Luna, M. E., Villalpando, C. Y., Martin, K. E., Carson, J. M., Johnson, A. E., Restrepo, C., and Roback, V. E., "Flight Testing a Real-Time Hazard Detection System for Safe Lunar Landing on the RocketPowered Morpheus Vehicle," AIAA 2015-0326, (2015).

[10] Stettner, R., "Compact 3-D Flash Lidar Video Cameras and Applications," Proceedings of SPIE, Vol. 7684, SPIE, Bellingham, WA, 768405-1 - 768405-8, (2010).

[11] Roback, V. E., Bulyshev, A. E., Amzajerdian, F., Brewster, P. F., Barnes, B. W., Kempton, K. S., and Reisse, R. A., "Helicopter Flight Test of a Compact, Real-Time 3-D Flash Lidar for Imaging Hazardous Terrain during Planetary Landing," AIAA 2013-5383, (2013). 